\title{
LA MESURE DU DIAMÈTRE DES BRINS DE LAINE
}

PAR

\author{
J. ROUGEOT \\ Station de Physiologie Animale, \\ Centre National de Recherches Zootechniques, Jouy-en-Josas (S.-et-O.)
}

\section{INTRODUCTION}

Dans les travaux de recherches sur la toison, il est souvent indispensable de connaître à un niveau déterminé et avec une grande précision, le diamètre de chacun des brins composant une mèche de laine.

En effet, si l'on veut suivre l'action de certains facteurs sur la croissance de la laine, il faut mesurer les diamètres à des niveaux déterminés de la mèche.

I1 est nécessaire également de connaitre les diamètres de chaque brin individuellement pour établir la courbe de fréquence de ces diamètres. En effet, on ne peut se contenter de la connaissance de la seule moyenne pour l'étude de la finesse de la toison.

En outre, l'établissement de ces courbes de fréquence, et le calcul de leurs paramètres, exigent la précision du micron dans la mesure des diamètres : cependant, il faut noter tout de suite, qu'une précision plus grande est tout à fait inutile puisque dans ce cas, on ferait intervenir dans les mesures le relief des écailles.

Enfin, la méthode de mesure doit être aussi rapide et commode que possible: l'opérateur doit pouvoir effectuer au moins 2 ooo mesures dans la journée, sans fatigue.

Ces conditions préliminaires nous obligent donc à écarter la méthode micrométrique, la méthode gravimétrique et 1'usage du micronaire ( $\left.{ }^{1}\right)$ (POHLE, I95I). En effet, la méthode micrométrique est trop lente et sa précision insuffisante ; les méthodes basées sur l'utilisation de la balance et du micronaire ne permettent pas d'obtenir les courbes de fréquence.

Au contraire, la méthode microscopique convient parfaitement puisque grâce à elle, on peut mesurer le diamètre des brins avec toute la précision voulue et établir les courbes de fréquence.

Pour obtenir une mesure parfaite de la. finesse il faudrait pouvoir mesurer l'aire de la section droite des brins de laine. En effet, la section droite des brins de laine n'est généralement pas circulaire; elle est sou-

(1) Micronaire, The Sheffield Corporation, Dayton, I, Ohio, U. S. A. 
vent plus ou moins elliptique, quelquefois même de forme polygonale, de telle sorte que la mesure d'un seul diamètre donne une idée imparfaite de la finesse de la laine.

C'est pourquoi de nombreux auteurs, Kronacher, SAxinger et Schaper (I925), Hardy (I933 et I935), Hardy et Wolf (I939), GrandSTAFF et HODDE (I940), de CUEACA (I952), se sont. efforcés de mettre au point des méthodes pour obtenir des coupes transversales des brins de laine. Malheureusement, malgré de notables améliorations apportées à la technique des sections transversales, les coupes obtenues ne sont point parfaites : de nombrenx brins sont coupés obliquement par rapport à 1'axe de la fibre et surtout, on ignore dans quelle mesure la forme et les dimensions de la section sont influencées par le tassement et par la contraction du milieu d'inclusion.

Aussi la mesure microscopique des diamètres selon le profil des brins, méthode la plus ancienne que DAUBEN'TON utilisa en I779, conserve-t-elle la faveur de nombreux chercheurs. D'ailleurs la Fédération Lainière Internationale a recommandé en 1948 cette méthode de mesure et fixé les règles à suivre, afin d'obtenir la meilleure estimation de la moyenne des diamètres.

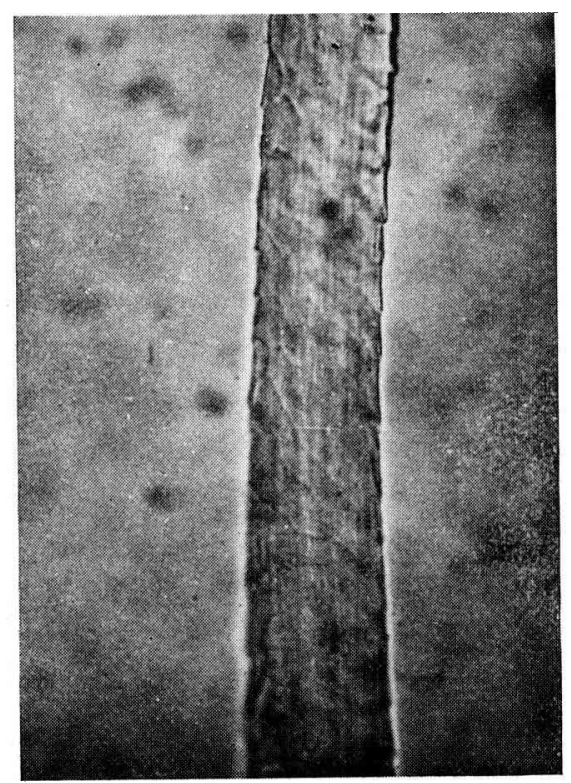

FIG. 1 .

Cependant, cette méthode exige de la part de 1'observateur tant de soin et d'attention, qu'elle est lente et très fatigante. C'est pourquoi nous avons cherché à rendre cette méthode plus rapide et plus aisée, 
tout en lui conservant sa précision. En effet, la difficulté des mesures provient notamment de la façon dont les brins de laine sont montés en vue de l'examen microscopique: les milieux de montages hydrophobes couramment utilisés pour examiner le brin entre lame et lamelle sous le microscope présentent des indices de réfraction de valeurs peu différentes de celui de la kératine ${ }^{1}$ ). Comme dans la grande majorité des cas 1a laine n'est pas pigmentée, l'image microscopique obtenue est à peine perceptible, et il en résulte une fatigue très rapide pour l'observateur. Aussi, pour rendre l'image du brin suffisamment visible est-on amené à réduire l'admission de la lumière. Cette pratique néfaste a pour conséquence l'apparition de franges à l'extérieur des bords de l'image (fig. I). La présence de ces franges augmente encore la difficulté des mesures (W.I.R. A., 1948).

On peut donc, théoriquement améliorer la méthode en montant les brins dans un milien dont l'indice de réfraction est nettement différent de celui de la kératine; nous obtiendrons ainsi une image du brin de laine très contrastée, aux contours très nets.

Aussi avons-nous songé à monter les brins de laine dans l'air. Cette idée n'est pas nouvelle, puisque A.-M. LARROY (I948) a déjà exposé une méthode de mesure microscopique où les brins de laine sont observés dans l'air: il étendait les brins entre deux bords d'un cadre de papier "bristol", et passait ainsi successivement chaque fibre sous 1'objectif du microscope. Malheureusement le procédé est trop lent. Nous proposons donc la méthode suivante qui élimine les inconvénients que nous venons de mentionner.

\section{TECHNIQUE}

\section{I $^{\circ}$ Lavage de la mèche}

La mèche de laine est lavée à fond, très soigneusement dans trois bains successifs d'un mélange d'éther sulfurique et d'alcool à parties égales. Chaque passage est suivi d'un essorage énergique entre les feuillets d'un matelas de papier filtre. En opérant ainsi les brins de laine conservent leurs positions relatives.

\section{$2^{\circ}$ Préparation des fragments des brins dont on mesure le diamètre}

I a mèche est coupée à un niveau déterminé en fragments de longueur voulue à l'aide des microtomes spéciaux de Hardy ou de Cuenca. Avant d'introduire la mèche dans la fente du microtome, il est

(1) Kératine $: \mathrm{n}=1,552$; baume du Canada $: \mathrm{n}=1,535$; huile de cêdre $: \mathrm{n}=1,515$. 
conseillé de garnir le fond de la fente d'un fragment de moelle de sureau. De même, avant d'introduire la languette de serrage du microtome, la mèche sera protégée par un autre fragment de moelle de sureau. Ainsi les brins seront serrés, sans crainte de détérioration.

Avant chaque coupe, on enduit les brins d'une solution sirupeuse de cellö̈dine dissoute dans un mélange d'acétone et d'acétate d'isoamyle. Ce liquide est un milieu d'inclusion parfait : il possède un pouvoir mouillant élevé, il sèche et durcit rapidement.

Quand l'enduit est sec, à l'aide d'une lame de rasoir, on découpe et on élimine soigneusement tout l'excès de l'enduit qui se trouve autour de la masse de laine.

Puis on effectue la coupe.

\section{$3^{\circ}$ Montage}

La coupe est déposée sur une lame de préparation microscopique rigoureusement propre. Les fragments de laine sont décollés les uns des

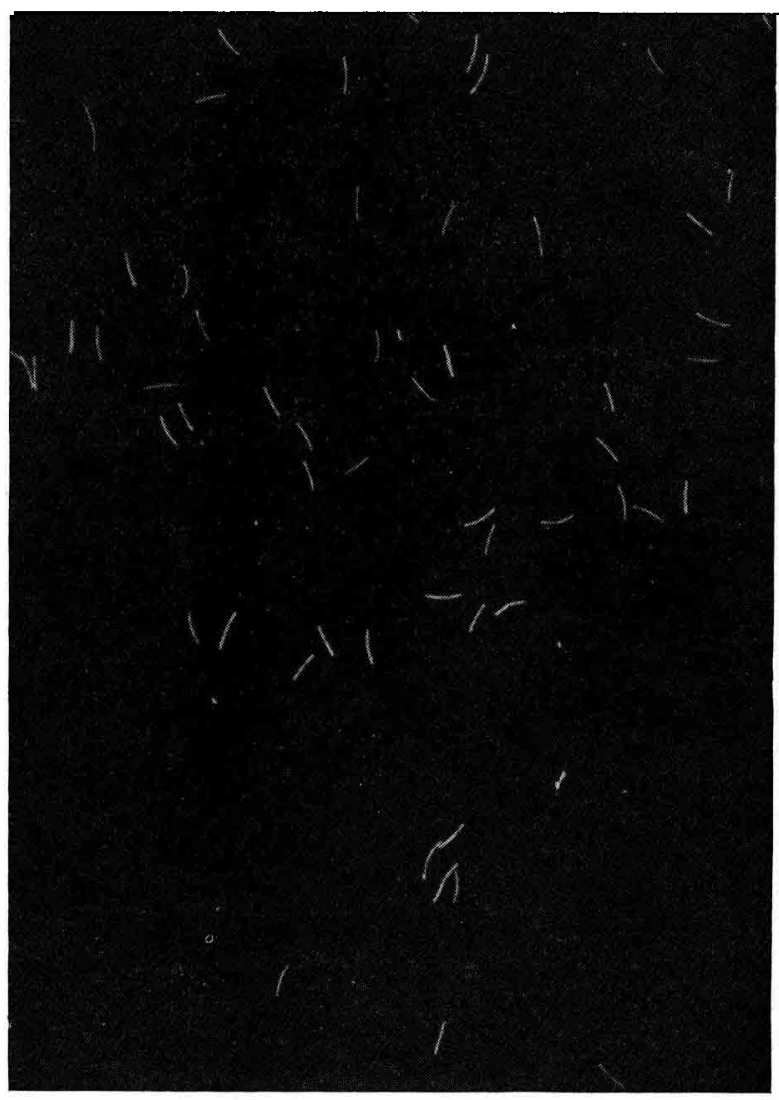

FIG. 2. 
autres, à l'aide d'une solution d'acétone et d'acétate d'isoamyle. Grâce à son pouvoir mouillant élevé, ce liquide s'étale rapidement sur toute la surface de la lame et provoque une dispersion très régulière des fragments de laine (fig. 2).

Il est indispensable d'éliminer tout l'enduit. Pour cela on laisse égoutter le solvant en excès, en tenant la lame légèrement inclinée de façon à éviter l'entraînement des particules de laine lorsque s'écoule le liquide.

On renouvelle plusieurs fois l'opération.

Le solvant restant s'évapore très rapidement et les fragments de laine sont collés sur la lame par une trace de celloïdine qui s'est maintenue par capillarité entre la surface du verre et la laine. En effet, en déplaçant les fragments de laine avec une fine aiguille de verre sous l'objectif du microscope nous avons pu déceler cette trace de vernis sous la forme d'une légère empreinte. Par contre, en dehors de la surface de contact du verre et de la laine il n'existe aucune trace d'enduit.

Nous avons ainsi conservé des préparations pendant plusieurs mois sans qu'aucun fragment de laine ne se détache.

\section{$4^{\circ}$ Mesure des diamètres au microscope}

La mesure s'effectue sur les fragments ainsi préparés à l'aide d'un microscope de projection.

L'image des fragments de laine grossie cinq cents fois est reçue sur un écran en verre dépoli et mesurée avec une règle graduée en plexiglass. La projection sur un écran opaque est à déconseiller car 1'observateur est gêné sérieusement dans ses mesures par l'ombre de sa tête ou de ses mains.

Nous conseillons de ne pas utiliser un objectif puissant afin d'obtenir une profondeur de champ suffisante. En ce qui nous concerne, nous nous sommes servis de l'objectif 5 et de l'oculaire compensateur 4 de STIASSNIE.

Dans ces conditions, la distance entre l'oculaire et l'écran est de 60 à $65 \mathrm{~cm}$, et nécessite donc l'emploi de télécommandes pour le chariot et la vis micrométrique.

\section{RÉSULTATS ET DISCUSSION}

Cette technique permet d'obtenir des brins de laine facilement décelables puisqu'ils présentent sur leurs bords deux zones obscures très larges dues à la réfraction des rayons lumineux (fig. 3). Le repérage est donc immédiat. Au contraire quand les brins sont montés selon la 
méthode habituelle il faut déplacer la préparation avec une lenteur extrême et observer soigneusement l'écran pour ne point laisser passer de brins.

On ne rencontre pas d'enchevêtrement de fragments de laine puisqu'on peut régler la dispersion en étalant les fragments sur une surface plus ou moins importante.

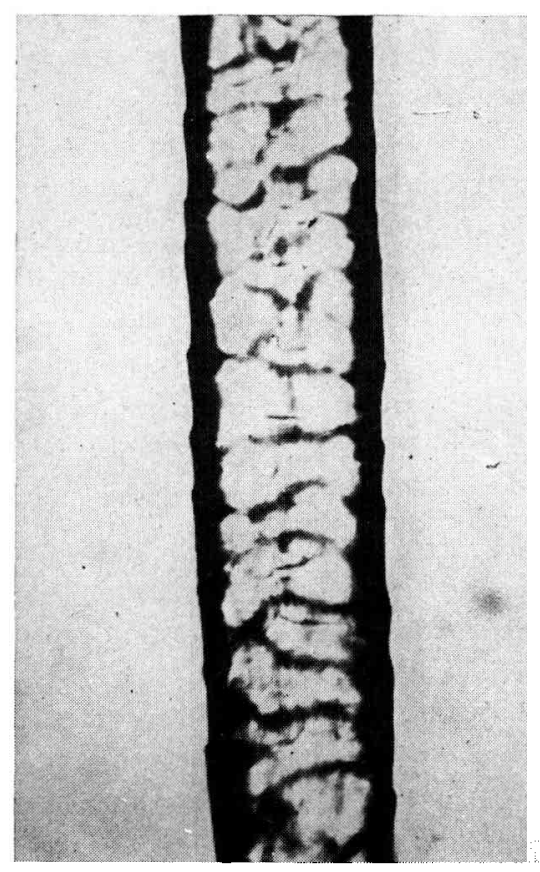

Fig. 3.

Einfin, les bords des brins sont très nets sans franges de Becke; la mise au point est rapide et facile.

Trois points doivent retenir particulièrement notre attention.

I. Le contraste et la netteté des contours de l'image des fragments de laine ne peuvent-ils être obtenus que par le montage de ces fragments dans l'air ?

2. Dans quelle mesure doit-on tenir compte de l'action de la température et surtout du degré hygrométrique de l'atmosphère dans la mesure des diamètres des brins de laine montés dans l'air ?

3. Peut-on rendre compte par la simple mesure des diamètres, selon le profil des brins, de l'ellipticité de leur section droite?

I. On pourrait employer des milieux de montage dont l'indice de réfraction présente une nette différence avec celui de la laine : huile de paraffine $(n=\mathbf{I}, 45)$, Aroclor $(n=\mathbf{I}, 63)$ ou Hyrax $(n=\mathbf{I}, 7)$. Or même 
avec ces produits on n'obtient pas des images satisfaisantes, les manipulations sont plus longues et les préparations sont plus délicates à manier, surtout si le montage est fait dans l'huile de paraffine.

Le microscope à contraste de phase ne nous donna pas d'image satisfaisante non plus. En effet, dans tous les microscopes à contraste de phase, un diaphragme placé dans le condensateur introduit des conditions d'éclairage plus voisines de l'éclairage cohérent, que de l'éclairage incohérent, d'où l'apparition de franges de diffraction inévitables.

2. Les brins de laine étant mesurés à l'air libre, il est nécessaire d'opérer dans un local dont 1'atmosphère soit conditionnée (ANDERson et PALMEE, I948). Cette condition peut gêner certains 1aboratoires.

C'est pourquoi nous avons étudié l'influence de l'humidité sur le diamètre des brins de laine à $20^{\circ} \mathrm{C}$ entre $40 \%$ et $85 \%$ d'humidité relative sur I ooo brins de laine, sur chacun desquels un diamètre avait été soigneusement repéré.

Les diamètres varient de façon notable seulement à partir de $60 \%$ $\mathrm{HR}$ : entre $60 \%$ et $82 \% \mathrm{HR}$ le diamètre augmente de $4 \%$. Par contre de $40 \%$ à $60 \%$ HR le diamètre n'augmente que de $0,6 \%$. Si bien qu'en opérant à $20^{\circ} \mathrm{C} \pm 0,2$ et à $55 \% \mathrm{HR} \pm 5 \%$, les erreurs de mesures dues à la variation du degré hygrométrique sont négligeables. Or des installations très simples et peu onéreuses, maintiennent facilement 1'atmosphère d'un local dans ces limites de température et d'humidité relative.

3. Les diamètres sont mesurés selon le profil du brin. Or nous savons que la section du brin de laine n'est pas toujours circulaire. Avec les brins montés dans 1'huile de cèdre, Anderson et PaLMEer (I947) ont montré que pour obtenir la moyenne réelle des diamètres il fallait opérer sur des fragments de laine d'au moins $0,8 \mathrm{~mm}$ de long: en effet, ces auteurs ont constaté que plus les fragments de laine étaient courts, plus la moyenne de diamètres mesurés sur ces fragments était élevée; c'est pourquoi ils émirent l'hypothèse que les fragments courts, ne présentant aucune ondulation, se plaçaient à plat entre la lame et la lamelle, le grand axe de leur section étant parallèle aux plans de celles-ci. Au contraire, quand la longueur des fragments était supérieure à $0,8 \mathrm{~mm}$, ils présentaient un diamètre quelconque à l'observation, et de cette manière on obtenait une meilleure estimation de la moyenne des diamètres. Nous avons confirmé l'hypothèse de PALMER et ANDERSon de la façon suivante: nous avons retourné sous l'objectif du microscope des fragments de laine provenant d'une mèche de toison de Brebis limousine et montés selon la technique décrite précédemment. Nous avons constaté que les fragments rectilignes reposaient tous sur la surface de la lame selon leur plus grand diametre. Par contre tous les fragments 
en arc de cercle présentaient de profil leur plus petit diamètre au niveau où la courbure était la plus accentuée.

On pourrait alors songer à obtenir l'indice d'ellipticité en comparant les moyennes obtenues à partir de mesures de diamètres faites sur des nombres égaux de fragments courbes et de fragments rectilignes. Malheureusement les populations de fragments courbes et de fragments rectilignes ne sont point comparables. En effet, les mèches de laine sont souvent très hétérogènes : elles se composent de diverses populations de brins qui diffèrent les unes des autres par la longueur, la finesse, la forme de la section droite, les ondulations des brins. En coupant donc tous les brins de laine à une même longueur, la répartition en fragments rectilignes et en fragments courbes ne se fait pas au hasard : en particulier, parmi les fragments rectilignes nous trouverons en majorité ceux qui proviennent de fibres raides, grossières, à section droite très elliptique. De fait dans la mèche précédemment étudiée les fragments rectilignes ont une section droite nettement plus elliptique que les fragments courbes: $\frac{a}{b}=\mathrm{I}, 32$ pour les rectilignes, et $\frac{a}{b}=\mathrm{I}, 2 \mathrm{I}$ pour les courbes. Cependant, à défaut d'indice d'ellipticité nous proposons un " coefficient de contour " déterminé de la façon suivante : on effectue les mesures des diamètres sur deux coupes successives. La première coupe est faite à $0, I \mathrm{~mm}$ et donne une préparation où tous les brins sont rectilignes. Cette préparation nous donnera donc la moyenne des grands diamètres: $\bar{x}_{\mathrm{m}}$. La deuxième préparation ne comprenant que des fragments d'au moins $0,8 \mathrm{~mm}$ de long nous donnera la moyenne des diamètres mesurés au hasard. Le " coefficient de contour " est défini par le. rapport des deux moyennes $\mathrm{C}=\frac{\bar{x}_{\mathrm{M}}}{\bar{x}}$. Le " coefficient de contour " permet de mettre en valeur l'ellipticité et d'attribuer une valeur quantitative à l'irrégularité de la forme de la section droite des brins de laine.

La possibilité de mesurer séparément les grands diamètres permet de faire également des remarques intéressantes sur les courbes de fréquence des mesures des diamètres : en effet la comparaison des histogrammes des mesures des grands diamètres et des petits diamètres montre des différences très nettes. L'histogramme des grands diamètres (graphique I) présente deux modes bien distincts. Il traduit le fait que dans la mèche étudiée il existe deux populations de brins; par contre l'histogramme des petits diamètres (graphique. II) est très étroit et ne présente qu'un seul mode. La population des petits diamètres est donc très homogène. Ėn effet, il n'existe pas de différence significative entre la moyenne $\bar{x}_{c}=30,1 \mu \pm 0,7$ des petits diamètres des brins courbes et la moyenne $\bar{x}_{r}=28$, I $\mu \pm 0,6$ des petits diamètres des brins rectilignes qu'on a retournés expérimentalement. De fait, la mèche 
se compose de deux sortes de fibres: les unes de section droite peu elliptique, dont 1e diamètre moyen est de 27-32 de section très elliptique, dont le petit diamètre est de $30-35 \mu$ et le grand de 50-55 $\ldots$. Or, comme il est de règle que les fibres dont les sections droites sont les plus elliptiques se trouvent parmi les plus grossières, la

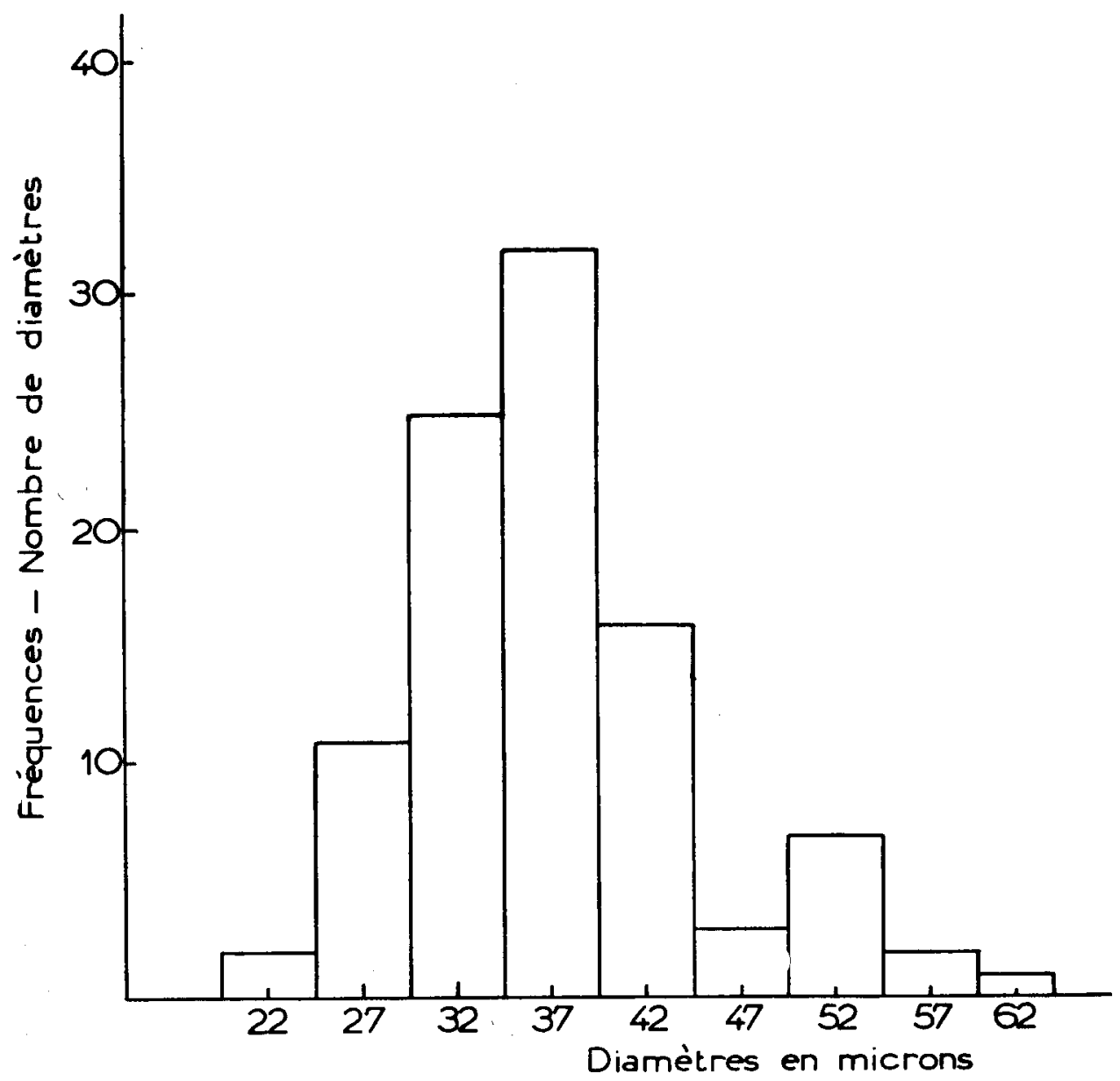

Graphique. I.

mesure des grands diamètres nous permet de distinguer les diverses populations de fibres d'une mèche. Ces renseignements ne nous sont point donnés, ni par la mesure des petits diamètres, ni par celle des diamètres mesurés au hasard. En effet, dans ce dernier cas la courbe de fréquence ne présente qu'un set1 mode et l'interprétation de la dissymétrie de la courbe obtenue est difficile.

D'autre part, en mesurant au hasard les diamètres des brins dont les sections droites sont elliptiques on obtient une moyenne des dia 
mètres inférieure à celle obtenue en ne mesurant que les grands diamètres. Or l'ellipticité prononcée des sections droites des fibres est un défaut (BARKFR, I93I). Mesurer les dianètres an hasard revient donc à amoin-

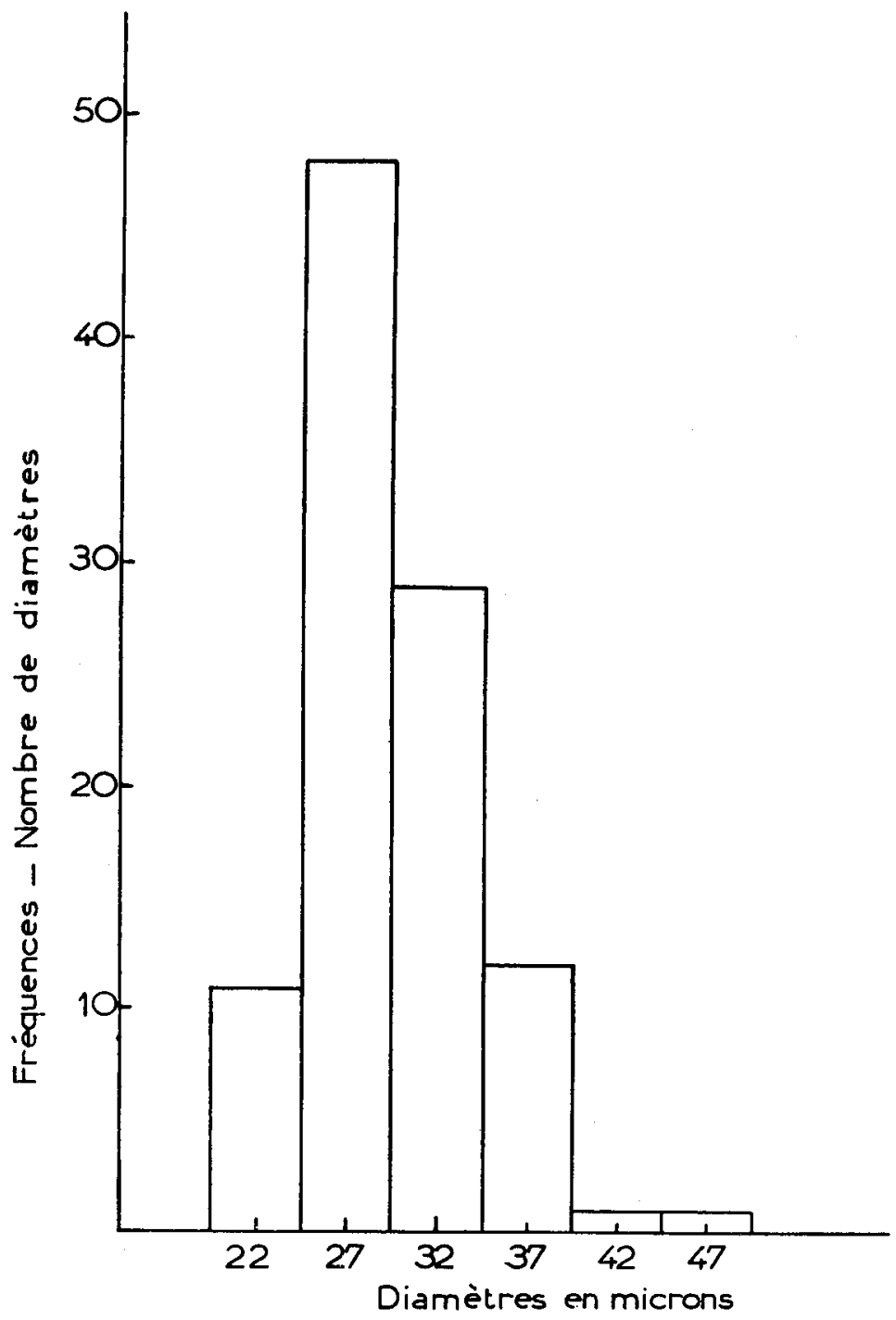

Graphique. II.

drir l'importance de ce défaut, ce qui ne semble pas raisonnable. C'est pourquoi la mesure de fragments rectilignes est importante, aussi bien pour la détermination de la moyenne des diamètres que pour l'étude de la dispersion de ces diamètres.

Enfin, pour terminer, une remarque nous permettra de distinguer 
par simple examen de l'image microscopique si un brin est nettement elliptique ou à peu près rond. En faisant la mise au point sur la partie supérieure du fragment, si sa section droite est circulaire les bandes latérales obscures de réfraction seront larges et les bords du brin flous (fig. 4). Au contraire, si le brin a une section droite elliptique, le grand̉ diamètre de la section étant parallèle au plan de l'écran, les bandes obscures seront très étroites et les bords du brin nets (fig. 5). Pour faire des observations aisées il faut régler le microscope à l'aide d'une préparation où les brins de laine sont remplacés par de fines aiguilles de verre

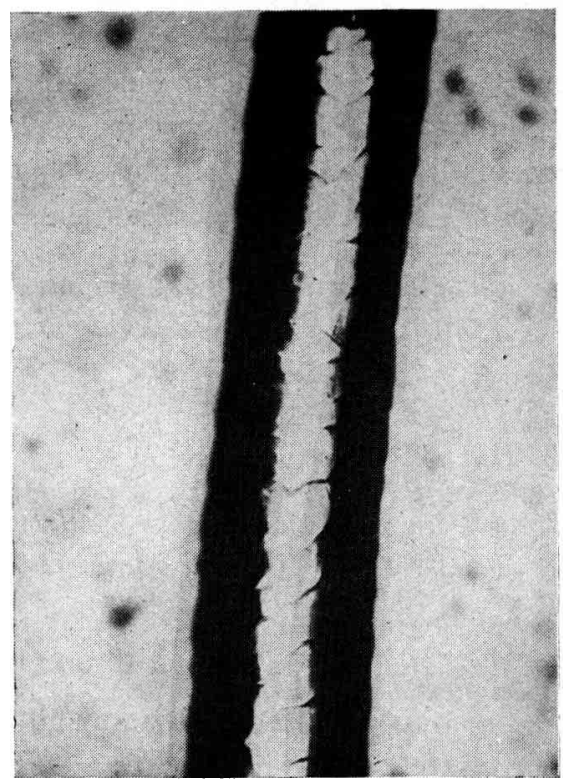

FIG. 4.

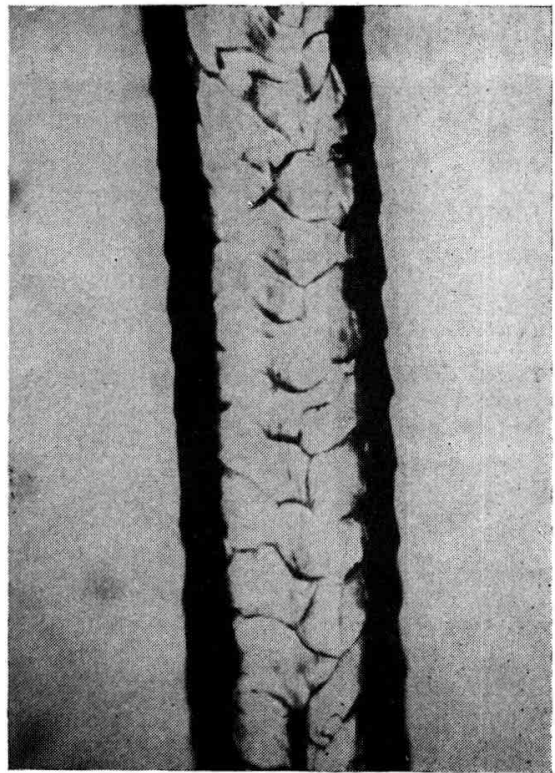

Fig. 5.

cylindriques. On effectue la mise au point sur les aiguilles de verre de manière que les bandes obscures latérales soient aussi larges que la bande lumineuse centrale.

Ces remarques peuvent être précieuses pour l'interprétation des résultats des mesures.

\section{RÉSUME}

L'étuc'e critique des méthodes de mesures des diamètres des brins de laine nous a permis de montrer que seule la méthode de mesure microscopique des diamètres selon le profil de la fibre donne de bons résultats.

Pour faciliter les mesures nous proposons de monter les fragments de laine dans 1'air grâce à un collage préalable sur une lame, sans lamelle : les fragments de laine sont alors très facilement décelables et ont des 
contours très nets. L'observateur n'est donc plus sujet à une fatigue rapide comme c'est le cas lorsque les brins sont montés dans un milieu dont l'indice de réfraction est voisin de celui de la laine. Les opérations sont également plus rapides.

L'erreur relative faite sur les mesures en travaillant dans une atmosphère dont la température est maintenue à $20^{\circ} \mathrm{C}$ et le degré hygrométrique à $55 \% \mathrm{HR} \pm 5 \%$ est inférieure à $0,6 \%$.

En opérant sur des fragments rectilignes, on ne mesure que les grands diamètres des sections droites. $\mathrm{I}_{4}$ a mesure des grands diamètres permet une étude plus précise de la répartition des différentes sortes de brins dans une mèche que ne le permet la mesure des diamètres faite au hasard. La mesure des grands diamètres combinée à la mesure des diamètres faite au hasard permet de calculer un " coefficient de contour " qui rend compte de l'ellipticité des sections droites des fibres. Enfin la moyenne des grands diamètres est une donnée intéressante en elle-même.

Ce travail a été effectué grâce à une subvention accordée par le Comité National Interprofessionnel de la laine à qui nous adressons nos vits remerciements.

\section{BIBLIOGRAPHIE}

ANderson (S. L.), PALMER (R. C.). - Effect of non circular cross-section on fibre diameter measurement of wool by the profil method. I.W.T.O. Tech. Ctte Proc., 1, 29-3I, I947.

Anderson (S. I.), Palmer (R. C.). - Effect of moisture on wool fineness measurement. I.W.T.O. Tech. Ctte. Proc., 2, 5-I2, 1948.

BARker (S. G.). - Wool quality. H. M. Stationery Office, I vol., I93I.

CUENCA (C. L. de). - Un microtome pour analyser la finesse des fibres de laine en coupe transversale. Bull. Inst. Text. France, 33, 59-62, I952.

Daubenton. - Mémoire sur les laines de France comparées aux laines étrangères. Histoire Acad. Roy. Sci. Mémoires, I-II, I779.

GrandstafF (J. O.), HodDe (W. I.). - A rapid method for projecting and measuring cross sections of wool fibres. U.S. Dept. A gri. Cir., 590, I I p., I940.

Hardy (J. I.). - Determination of fibre fineness. A rapid method using a new cross sectionning device. Textile Res., 3, 381-387, I933.

HaRdy (J. I.). - A practical laboratory method of making thin cross sections of fibres. U.S. Dept. Agri. Cir., 378, II p., I935.

HARdy (J. I.), WOLF (H. W.). - Two rapid methods for estimating fineness and cross sectional variability of wool. U. S. Dept. Agri. Cir., 543, I6 p., I939.

Kronacher (C.), Saxinger (G.), Schäper (W.). - Die Wollefeinheitsbestimmung an Querschnitt im Projektionbild. Z. Tierz. Züchtungsbiol., 4, 2I3-253, I925.

LEROY (A. M.). - Le Mouton. Librairie Hachette. I vol., I948.

POHLE (E. M.). - Application of the micronaire for measuring fineness of wool. U. S. Dept. Agri. P. M. A. Livestock Branch Report, I95I.

Wool Industries Research Association. - Detailed description of the projection methods for the measurement of fibre diameter to be used in the I948-9 experiments. I. W. T. O. Tech. Cttee. Proc., 2, 22-26, 1948. 